\title{
Threshold behavior and analytical fitting of partial wave capture probabilities for attractive $R^{-n}$ potentials
}

\author{
E. I. Dashevskaya, ${ }^{a b}$ I. Litvin, ${ }^{a b}$ E. E. Nikitin ${ }^{a b}$ and J. Troe ${ }^{* b c}$ \\ Received 26th June 2009, Accepted 28th July 2009 \\ First published as an Advance Article on the web 21st August 2009 \\ DOI: $10.1039 / \mathrm{b} 912679$ a
}

Numerically accurate analytical fittings for partial wave capture probabilities in the field of $R^{-n}$ potentials ( $n=4$ and 6 ) are presented across practically interesting ranges of probabilities. The results demonstrate the performance of the Bethe and Wigner threshold laws at low collision energies and should be useful for practical applications.

\section{Introduction}

Cross sections and rate coefficients for complex-forming encounters between two particles under the action of isotropic attractive interparticle potentials are completely determined by capture probabilities $P_{\ell}$. They correspond to the close approach of the partners to an absorbing complex surface in a particular channel with the orbital momentum quantum number $\ell$, and the set of $P_{\ell}$ determines the partial and total complex-forming cross sections $\sigma_{\ell}$ and $\sigma$ through

$$
\sigma(k)=\sum_{\ell=0}^{\infty} \sigma_{\ell}(k), \quad \sigma_{\ell}(k)=\frac{\pi(2 \ell+1)}{k^{2}} P_{\ell}(k)
$$

Here $k$ is the wave vector of the relative motion of the partners. For complete capture, the probabilities $P_{\ell}(k)$ are identified with the probabilities $T_{\ell}(k)$ of transmission either above the potential drop for the channel with $\ell=0$ or through the potential barrier created by the attractive potential and the repulsive centrifugal potential for $\ell>0$. For potentials of the form $U_{n}(R)=-\mathrm{c}_{n} / R^{n}, c_{n}>0$ and the additional assumption that the relative motion of the partners near the complex surface is quasiclassical, the numerical quantities $T_{\ell}^{(n)}(k)=P_{\ell}^{(n)}(k)$ are well known ${ }^{1}$ up to $\ell_{\max }$ that is large enough such that $P_{\ell}^{(n)}(k)$ with $\ell>\ell_{\max }$ can be well approximated by classical step functions of the energy. As was shown in ref. 1 the accurate capture probabilities $P_{\ell}^{(n)}$ depend only on the reduced wave vector $\kappa_{n}=R_{n} k$ where $R_{n}$ are characteristic lengths of the potentials $U_{n}$ that mark the position of the region where the reflection occurs. For $n=4$ and $n=6$, these quantities are $R_{4}=\left(2 \mu c_{4} / \hbar^{2}\right)^{1 / 2}$ and $R_{6}=\left(2 \mu c_{6} / \hbar^{2}\right)^{1 / 4} \cdot{ }^{2}$

In practical applications, often the need arises to use the functions $P_{\ell}^{(n)}(k)$ in approximate analytical form and to understand the applicability of their threshold energy dependence. In particular, for $n=4$, some analytical representations of $P_{\ell}(k)$ were suggested, in ref. 1,2 for $\ell=0$ and in ref. 3 for $\ell=1,2,3$, and 4 . These analytical expressions were designed only by consideration of their accuracy and without resorting

\footnotetext{
${ }^{a}$ Schulich Faculty of Chemistry, Technion-Israel Institute of Technology, Haifa, 32000, Israel

${ }^{b}$ Max-Planck-Institut für Biophysikalische Chemie, Am Fassberg 11, Göttingen D-37077, Germany.E-mail: shoff@gwdg.de

${ }^{c}$ Institut für Physikalische Chemie, Universität Göttingen,

Tammannstrasse 6, Göttingen D-37077, Germany
}

to basic criteria. In this note, we return to this question for the practically interesting cases $n=4$ and $n=6$ and we suggest approximate analytical expressions $P_{\ell}^{(n) \text {,app }}(k)$ that are forced to comply with the known properties of the accurate probabilities $P_{\ell}^{(n)}(k)$.

The properties of the probabilities, which we take into account in constructing $P_{\ell}^{(n) \text {,app }}(k)$, are given in the following.

(i) The approximate probabilities should approach the Bethe-Wigner dependences of the probabilities on the wave vector $k$ for small $k$ which are given by ref. 4

$$
\left.P_{\ell}^{(n), \text { app }}(k)\right|_{\text {small } k}=\left.P_{\ell}^{(n)}(k)\right|_{\text {small } k}=C_{\ell}^{(n)}\left(R_{n} k\right)^{2 \ell+1}
$$

The coefficients $C_{\ell}^{(n)}$ can be found from the solution of the Schroedinger equation at zero energy, and are known in analytical form, ${ }^{5}$ see below.

For the particular case $\ell=0$, when written through the imaginary part of the scattering length $a^{\prime \prime}$, the partial cross sections can be represented in the more extended form [ref. 4, Sect.143]

$$
\left.\sigma_{0}(k)\right|_{\text {small } k}=\frac{4 \pi\left|a^{\prime \prime}\right|}{k}\left(1-2\left|a^{\prime \prime}\right| k\right)
$$

The comparison of eqn (3) and eqn (1) leads to the representation of the probability through second order in $k$ :

$$
\begin{aligned}
\left.P_{0}^{\text {app }}(k)\right|_{\text {small } k} & =\left.P_{0}(k)\right|_{\text {small } k} \\
& =4\left|a^{\prime \prime}\right| k-8\left|a^{\prime \prime}\right|^{2} k^{2}+O\left(k^{3}\right)
\end{aligned}
$$

(ii) The approximate and accurate probabilities should coincide at that value $k=k_{\ell}^{(1 / 2)}$ where the accurate probabilities are equal to $1 / 2$ :

$$
\left.P_{\ell}^{\mathrm{app}}(k)\right|_{k=k_{\ell}^{(1 / 2)}}=\left.P_{\ell}(k)\right|_{k=k_{\ell}^{(1 / 2)}}=1 / 2
$$

(iii) The approximate probabilities should have the correct asymptotic behavior at large $k$,

$$
\left.P_{\ell}^{(n), \text { app }}(k)\right|_{\text {large } k},\left.P_{\ell}^{(n)}(k)\right|_{\text {large } k} \rightarrow 1
$$

In connection with the condition (i) we note that the linear dependence of the s-wave capture probability on $k$ and the super-linear dependence for higher momenta are due to different physical effects, the partial transmission of the incoming wave above the drop of the potential for $\ell=0$ (Bethe law ${ }^{6}$ ) and the passage of the incoming waves through 
the centrifugal barriers for $\ell>0$ (Wigner law ${ }^{7}$ ). However, as remarked in ref. 8 , the Wigner threshold law can be formally extended down to $\ell=0$ in such a way that the coefficients $C_{\ell}$ for $\ell \geq 0$ are given by a unified expression (see ref. 5 and the review ${ }^{8}$ ),

$$
C_{\ell}^{(n)}=\frac{4 \pi^{2}}{2^{2 \mu}(n-2)^{2 \nu} \mu \nu[\Gamma(\mu) \Gamma(\nu)]^{2}}
$$

with $\mu=\ell+1 / 2, \nu=(2 \ell+1) /(n-2)$.

In what follows, we consider the case $n=4$ in some detail (Section 2), and the case $n=6$ in shorter form (Section 3), since the latter it basically similar to $n=4$. For simplicity, we also omit super- and sub-scripts in $P_{\ell}^{(n)}$ and $\kappa_{n}$ once the context permits. Our results are summarized in Section 4.

\section{Capture of charged particles by isotropically polarizable neutral molecules $(n=4)$}

2a. s-Wave capture $(\ell=0)$

Within the considered capture model, $P_{0}$ is identified with the transmission probability $T_{0}$ across the potential drop. The latter quantity is obtained from eqn (2) and (7) as $\left.T_{0}(\kappa)\right|_{\kappa \rightarrow 0}=4 \kappa$. eqn (4) then implies $\left|a^{\prime \prime}\right|=R_{4}$ such that $P_{0}(\kappa)$ for small $\kappa$ can be written as

$$
\left.P_{0}(\kappa)\right|_{\kappa \ll 1}=4 \kappa-8 \kappa^{2}+O\left(\kappa^{3}\right)
$$

A function that satisfies eqn (2)-(4) may, e.g., be chosen in the form

$$
P_{0}^{\mathrm{app}}(\kappa)=1-A \exp (-\alpha \kappa)-B \exp (-\beta \kappa)
$$

with four fitting parameters, $A, \alpha, B$, and $\beta$. These should be determined from the conditions

$$
\begin{aligned}
\left.P_{0}^{\mathrm{app}}(\kappa)\right|_{\kappa=0} & =0, \\
\mathrm{~d} P_{0}^{\mathrm{app}}(\kappa) /\left.\mathrm{d} \kappa\right|_{\kappa=0} & =4 \\
\mathrm{~d}^{2} P_{0}^{\mathrm{app}}(\kappa) /\left.\mathrm{d} \kappa^{2}\right|_{\kappa=0} & =16 \\
P_{0}^{\mathrm{app}}\left(\kappa_{0}^{(1 / 2)}\right) & =1 / 2
\end{aligned}
$$

Here, $\kappa_{0}^{(1 / 2)}=0.1836$ is derived from the equation $P_{0}\left(\kappa_{0}^{(1 / 2)}\right)=1 / 2$ where $P_{0}(\kappa)$ is determined numerically. Rather unexpectedly we found that the form of the analytical expression in eqn (9) with four fitting parameters is not flexible enough to satisfy all four conditions of the eqn (10). We, therefore, determined the values of $A, \alpha, B$, and $\beta$ from eqn (10a), (10b) and (10d) requiring a minimum deviation of $\mathrm{d}^{2} P_{0}^{\mathrm{app}}(\kappa) /\left.\mathrm{d} \kappa^{2}\right|_{\kappa=0}$ from the value of 16 as given in eqn (10c). In particular, this fitting yields the DLNT (acronym for the authors of ref. 3) capture probability in the form

$$
P_{0}^{\text {DLNT }}=1-0.25 \exp (-1.41 \kappa)-0.75 \exp (-4.86 \kappa)
$$

(In our previous work ${ }^{3}$ we have also used the simpler forms $P_{0} \approx 1-0.5 \exp (-2 \kappa)-0.5 \exp (-6 \kappa)$ or $P_{0} \approx 1-0.25$ $\exp (-1.387 \kappa)-0.75 \exp (-4.871 \kappa)$ which are slightly less accurate than eqn (11)). Another form for $P_{0}^{\text {app }}(\kappa)$ was discussed by Quack and Troe ${ }^{9}$ for transmission above a truncated Morse potential, called also the Woods-Saxon step potential and elaborated also in ref. 4 and 8 . When the height of the potential is large (which is the case here), this potential approaches an attractive exponential potential for which the transmission coefficient assumes the form

$$
T(k)=1-\exp (-\pi L k)
$$

where $L$ is the characteristic length parameter. By choosing $L$ from the condition that eqn (12) satisfies the small $\kappa$ expansion (see eqn (8)), one gets an interpolation formula valid across the whole range of the collision energies. This was later recognized by $\mathrm{Klots}^{2}$ who used the expression (12) by choosing $\mathrm{L}$ from the condition that the capture probability should be consistent with the Vogt-Wannier limit ${ }^{10}$ of the s-wave capture cross section. In this way, the Quack-Troe-Klots (QTK) expression for the capture probability assumes the form (see also ref. 8)

$$
P_{0}^{\mathrm{QTK}}(\kappa)=1-\exp (-4 \kappa)
$$

Interestingly, eqn (13) can be also regarded as a particular case of eqn (9) whose parameters are determined from eqn (10a)-(10c), ignoring eqn (10d). From the above, we expect that the DLNT approximation (eqn (11)) will be superior to the QTK approximation (eqn (13)) for noticeable probabilities (since the DLNT approximation satisfies the condition (10d) while the QTK approximation does not)), but the reverse will be true for small probabilities (since the QTK approximation, being derived from the accurate solution of the transmission problem, satisfies the three conditions (10a), (10b) and (10c), while DLNT does not satisfy condition (10c), yielding 18 instead of 16). This is illustrated by Fig. 1 which shows the ratios $P_{0}^{\mathrm{DLNT}}(\kappa) / P_{0}(\kappa)$ and $P_{0}^{\mathrm{QTK}}(\kappa) / P_{0}(\kappa)$. Note that the maximum deviation of the ratio $P_{0}^{\mathrm{QTK}}(\kappa) / P_{0}(\kappa)$ from unity is about $8 \%$ while $P_{0}^{\mathrm{DLNT}}(\kappa) / P_{0}(\kappa)$ does not deviate from unity by more than $0.8 \%$. Fig. 2 shows the ratio $P_{0}^{\mathrm{DLNT}}(\kappa) / P_{0}(\kappa)$ superimposed on the graph of the accurate

\begin{tabular}{|c|c|c|c|c|c|c|c|c|}
\hline$\ell$ & $C_{\ell}$ & $C_{\ell}^{*}$ & $C_{\ell}^{\mathrm{WKB}}$ & $\kappa_{\ell}^{(1 / 2)}$ & $\kappa_{\ell}^{*}$ & $a_{\ell}$ & $b_{\ell}$ & $c_{\ell}$ \\
\hline 1 & 0.444 & 0.45 & 0.553 & 1.187 & 0.68 & 1.5 & -0.5 & 0.1 \\
\hline 2 & $1.98 \times 10^{-3}$ & $2.2 \times 10^{-3}$ & $2.26 \times 10^{-3}$ & 3.186 & 2.12 & 0.89 & -0.138 & 0.018 \\
\hline 3 & $1.61 \times 10^{-6}$ & $2.0 \times 10^{-6}$ & $1.77 \times 10^{-6}$ & 6.189 & 4.95 & 0.63 & -0.055 & 0.004 \\
\hline 4 & $4.05 \times 10^{-10}$ & $6.0 \times 10^{-10}$ & $4.37 \times 10^{-10}$ & 10.185 & 9.14 & 0.46 & -0.02 & 0.003 \\
\hline
\end{tabular}
capture probability $P_{0}(\kappa)$ and its small- $k$ counterpart (eqn (8)) extrapolated up to the point where the relative deviation $\left(P_{0}-\left.P_{0}\right|_{\text {small }} \kappa\right) / P_{0}$ becomes well visible. This representation helps to visualize the regions of $\kappa$ which are of interest for applications.

\section{2b. Capture of higher waves $(\ell>0)$}

Approximate expressions for capture probabilities with $\ell>0$ have to be split into two parts, one for small $\kappa$ and one for $\kappa$

Table 1 Fitting parameters for $P_{\ell}^{\mathrm{DLNT}}(\kappa)$ in potentials with $n=4$ 


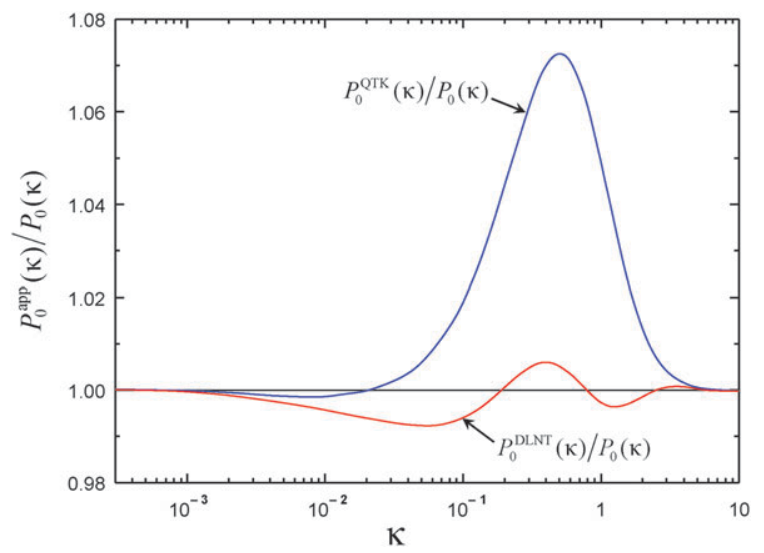

Fig. 1 Accuracy of the approximate capture probabilities $P_{0}^{\mathrm{QTK}}$ and $P_{0}^{\mathrm{DLNT}}$.

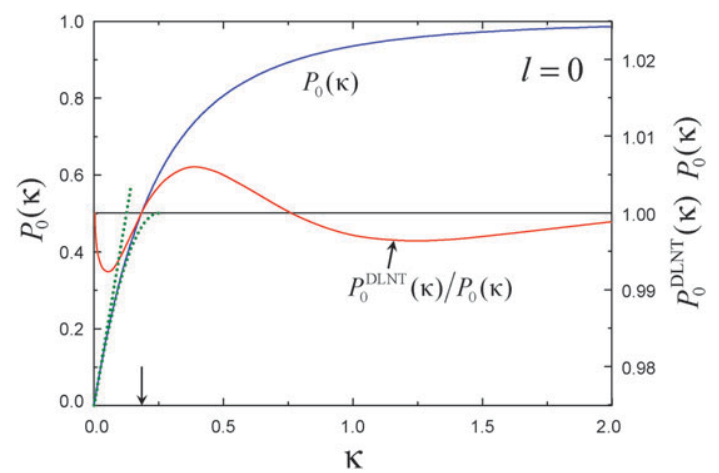

Fig. 2 Accuracy $P_{0}^{\mathrm{DLNT}}(\kappa) / P_{0}(\kappa)$ (right ordinate axis) and accurate capture probabilities $P_{0}(\kappa)$ (left ordinate axis). The arrow at the abscissa marks the value of $\kappa=\kappa_{0}^{(1 / 2)}$. The two dotted curves correspond to the linear and quadratic approximations in eqn (8).

that correspond to energies near to the maximum of the barrier.

In the standard interpretation of the Wigner law, the dependence $P_{\ell}(k) \propto k^{2 \ell+1}$ for small $k$ is attributed to the tunneling asymptotics of the wave function of free motion which at a distance $R_{m}$ matches with the wave function of the complex characterized by an "internal" short-range potential. ${ }^{4}$ In other words, the tunneling asymptotics terminates at $R=R_{m}$ with $R_{m}$ being independent from $\ell$. In our case, the tunneling asymptotic of the wave function of free motion determines the flux toward the complex surface after the wave leaves the centrifugal barrier and proceeds in the direction of decreasing interfragment distance. Thus, the tunneling asymptotics terminates somewhere at the internal part of the centrifugal barrier, at a position which depends on $\ell$. Under these circumstances, one would have expected deviations of the capture probabilities from the form of eqn (2). Nonetheless, it was found analytically, by solving the wave equation for zero energy, ${ }^{5}$ and numerically ${ }^{1}$ that transmission (i.e. capture) probabilities comply with eqn (2). In other words, the $\left.P_{\ell}(\kappa)\right|_{\kappa \ll \kappa_{\ell}^{(1 / 2)}}$ can be presented as

$$
\left.P_{\ell}(\kappa)\right|_{\kappa \ll \kappa_{\ell}^{(1 / 2)}}=C_{\ell} \kappa^{2 \ell+1}
$$

with the coefficients $C_{\ell}$ given by eqn (7) for $\mu=\nu=\ell+1 / 2$ (see Table 1). On the other hand, one can try to estimate small transition probabilities within the WKB approximation by "working with WKB waves far from the semiclassical limit". The standard WKB form of the small transmission probability through a centrifugal barrier reads

$$
P_{\ell}^{\mathrm{WKB}}(\kappa)=\exp \left(-2 \int_{\rho_{\min }}^{\rho_{\max }} \sqrt{(\ell+1 / 2)^{2} / \rho^{2}-1 / \rho^{4}-k^{2}} \mathrm{~d} \rho\right)
$$

where $\rho_{\min }$ and $\rho_{\max }$ are the roots of the radicand, and the centrifugal energy is written with the Langer modification, ${ }^{4}$ $\ell(\ell+1) \rightarrow(\ell+1 / 2)^{2}$. We have found that the quantities $C_{\ell}^{\mathrm{WKB}}$ defined as

$$
C_{\ell}^{\mathrm{WKB}}=P_{\ell}^{\mathrm{WKB}}(\kappa) / \kappa^{2 \ell+1}
$$

do not depend on $\kappa$ for $\kappa<0.1$ and agree with known analytical expression (see eqn (220) from ref. 8). As expected, ${ }^{8}$ $C^{\mathrm{WKB}}$ are larger than the accurate coefficients $C$, see Table 1 .

For medium and large $\kappa$, one can try to approximate the capture probability by the expression for the transmission probability across a parabolic barrier

$$
P_{\ell}(\kappa) \approx P_{\ell}^{\mathrm{par}}(\kappa)=\frac{1}{1+\exp \left(-2 H_{\ell}^{\mathrm{par}}(\kappa)\right)}
$$

with $2 H_{\ell}^{\mathrm{par}}(\kappa)=\left(\pi \sqrt{2} / \ell^{3 / 2}(\ell+1)^{3 / 2}\right)\left(\kappa^{2}-\kappa_{\ell}^{2}\right), \kappa_{\ell}=\ell(\ell+1) / 2$.

In our earlier work, ${ }^{1}$ we have found that the expression (17) is useful in constructing overall probabilities, but the accuracy of the approximation is not high enough, especially for low values of $\ell$ when the centrifugal barrier deviates strongly from the parabolic form near to its maximum $\kappa_{\ell}^{2} / 2$. We therefore modified the expression (16) writing

$$
P_{\ell}(\kappa) \approx g_{\ell}(\kappa)=\frac{1}{1+\exp \left(-2 H_{\ell}(\kappa)\right)}
$$

with a fitting monotonically-increasing function $H_{\ell}(\kappa)$ that vanishes at $\kappa=\kappa_{\ell}^{(1 / 2)}$ and guaranties the correct asymptotics $\left.g_{\ell}(\kappa)\right|_{\kappa \gg 1} \rightarrow 1$. (The function $g_{\ell}(\kappa)$ in eqn (18) is equivalent to the function $G_{\ell}(\kappa)$ introduced in the Appendix of ref. 3 provided that the misprinted term 0.5 is removed from the r.h.s. of the expression that defines $\left.G_{\ell}(\kappa)\right)$. With expression (18), the condition in eqn (5) is automatically satisfied, and the only remaining problem is a satisfactory matching of expressions in eqn (14) and (18) through a certain function $P_{\ell}^{\text {app }}$ at an intermediate point $\kappa=\kappa_{\ell}^{*}$. We have found that the matching is achieved by piece-wise definition of $P_{\ell}^{\mathrm{app}}(\kappa)$ whose explicit form, $P_{\ell}^{\mathrm{DLNT}}(\kappa)$, is

$$
P_{\ell}^{\mathrm{DLNT}}(\kappa)= \begin{cases}\left(\kappa / \kappa_{\ell}^{*}\right)^{2 \ell+1} g_{\ell}\left(\kappa_{\ell}^{*}\right), & \kappa \leq \kappa_{\ell}^{*} \\ g_{\ell}(\kappa), & \kappa \geq \kappa_{\ell}^{*}\end{cases}
$$

where $\kappa^{*} \ell$ is determined from the condition

$$
\left(k_{\ell}^{*}\right)^{-2 \ell-1} g\left(\kappa_{\ell}^{*}\right)=C_{\ell}
$$

The matching is reasonably smooth (though it contains a visible kink in the derivative) and the asymptotic behavior of $P_{\ell}^{\text {app }}(\kappa)$ is satisfactory when $H_{\ell}(\kappa)$ is taken as a linear function of 
$\left(\kappa-\kappa_{\ell}^{(1 / 2)}\right)$. If one accepts a more complicated form of $H_{\ell}(\kappa), v i z$ :

$H_{\ell}(\kappa)=a_{\ell}\left(\kappa-\kappa_{\ell}^{(1 / 2)}\right)+b_{\ell}\left(\kappa-\kappa_{\ell}^{(1 / 2)}\right)^{2}+c_{\ell}\left(\kappa-\kappa_{\ell}^{(1 / 2)}\right)^{3}$

and considers the matching position $\kappa^{*} \ell$ as a fitting parameter by relaxing the condition in eqn (14), then the analytical functions $P_{\ell}^{\mathrm{DLNT}}(\kappa)$ become quite close to the graphical representation of $P$ (see Fig. 1 of ref. 1). This procedure transforms the expression (14) into

$$
\left.P_{\ell}^{\mathrm{DLNT}}(\kappa)\right|_{\kappa \ll \kappa_{\ell}^{(1 / 2)}}=C_{\ell}^{*} \kappa^{2 \ell+1}
$$

with the coefficients $C_{\ell}^{*}$ listed in Table 1 . We note here that the relaxation of the constraint imposed by eqn (14) for $\ell>0$, is similar in spirit to the relaxation of the constraint by eqn (10c) for $\ell=0$. In both cases, tolerating an incorrect behavior of the small probability in the limit $\kappa \rightarrow 0$ allows one to achieve a better approximation in the region where the probability is noticeable. Fig. 3 shows the "accuracy" graphs, the ratio $P_{\ell}^{\mathrm{DLNT}}(\kappa) / P_{1}(\kappa)$ superimposed on the graphs of capture probabilities $P_{1}(\kappa)$. The kink in the derivative of now is seen in the "accuracy" graph which shows the overall accuracy to be better than about $2.5 \%$. The arrow marks the point $\kappa=\kappa_{1}^{(1 / 2)}$ where $P_{1}^{\text {DLNT }}=1 / 2$. The vertical dashed line separates the regions where $P_{1}^{\mathrm{DLNT}}(\kappa)$ is given either by the "Wigner" or by the "parabolic" expressions (the upper or lower lines in the r.h.s. of eqn (19)), and the dotted line corresponds to the Wigner threshold relation, eqn (14) as obtained from numerical calculations. Fig. 4-6 show similar graphs for $l=2-4$. One notices that the maximum deviations of $P_{\ell}^{\text {DLNT }}(\kappa) / P_{0}(\kappa)$ from unity increase with increasing $l$. However, they remain below $5 \%$ for $P_{l}>0.1$ and thus appear acceptable in practical applications.

The parameters $\kappa^{*}, \kappa_{\ell}^{(1 / 2)}, a_{\ell}, b_{\ell}$, and $c_{\ell}$ entering into eqn (19) and (21) are listed in Table 1. It turns out that the values of $\kappa_{\ell}^{(1 / 2)}$ are very close to the quantities $\kappa_{\ell}$, and the coefficients $a_{\ell}$ are close to the respective coefficient in the linear term of $H_{\ell}^{\text {par }}$. The comparison of correct and Wigner-type probabilities at small $\kappa$ is also displayed in Fig. 2-6.

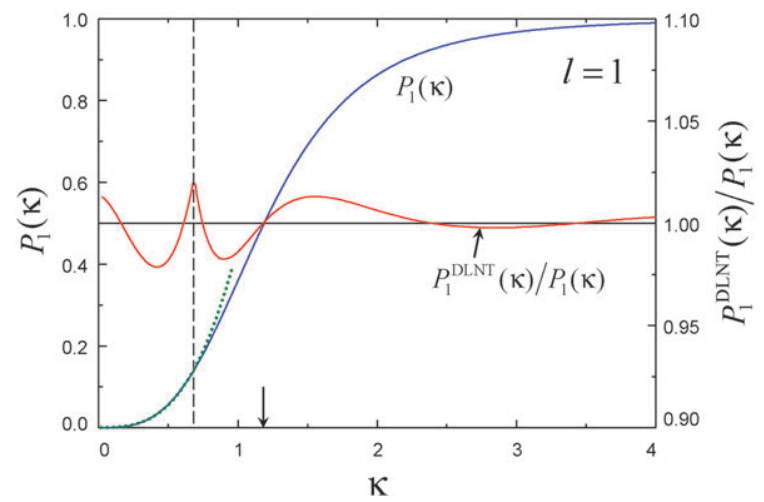

Fig. 3 Accuracy $P_{1}^{\mathrm{DLNT}}(\kappa) / P_{1}(\kappa)$ (right ordinate axis) and accurate capture probability $P_{1}(\kappa)$ (left ordinate axis). The arrow marks the point $\kappa=\kappa_{1}^{(1 / 2)}$ and the vertical dashed line at $\kappa=\kappa^{*}{ }_{1}$ separates the regions where $P_{1}^{\mathrm{DLNT}}(\kappa)$ is given by the upper or lower expressions in the r.h.s. of eqn (19). The dotted curve corresponds to the Wigner threshold relation, eqn (14).

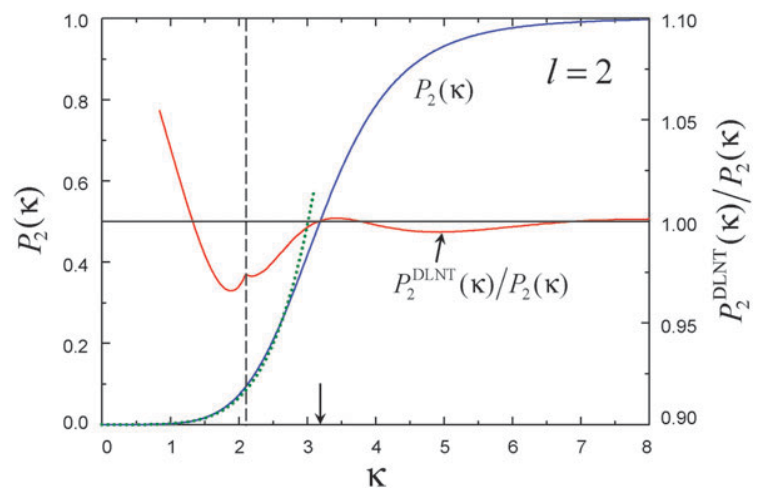

Fig. 4 As in Fig. 3, but for $\ell=2$.

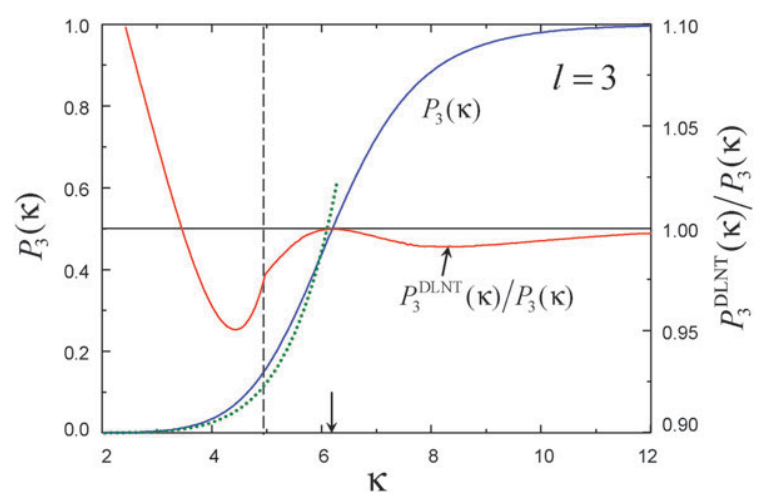

Fig. 5 As in Fig. 3, but for $\ell=3$.

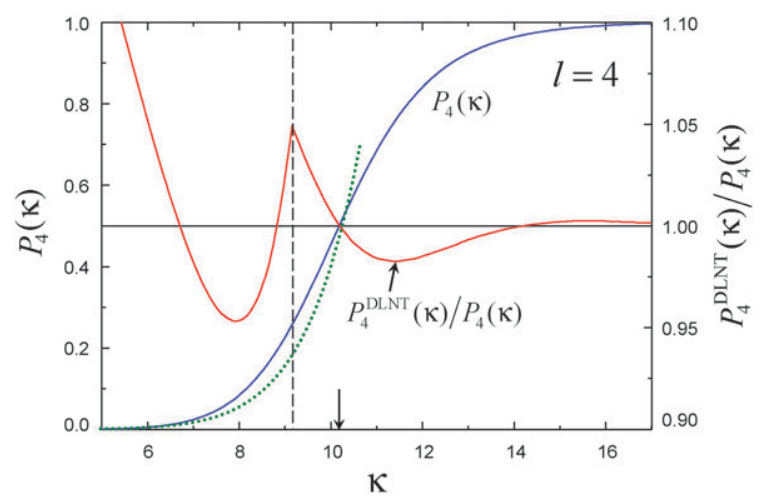

Fig. 6 As in Fig. 3, but for $\ell=4$.

\section{Capture of isotropically polarizable neutral molecules $(n=6)$}

This section essentially repeats the material of Section 2, but now for $n=6$. For $\ell=0$, the counterparts of eqn (11) and (13) read:

$$
\begin{aligned}
P_{0}^{\mathrm{DLNT}}(\kappa)= & 1-0.9312 \exp (-2.0165 \kappa) \\
& -0.0688 \exp (-0.500 \kappa) \\
P_{0}^{\mathrm{QTK}}(\kappa)= & 1-\exp (-1.912 \kappa)
\end{aligned}
$$

The accuracy of the DLNT approximation in eqn (23) is about $0.5 \%$. The maximum deviation of QTK approximation in eqn (24) from the accurate probability is about $3 \%$. (see Fig. 7 and 8). Compared to the previous case $n=4$, the 
Table 2 Fitting parameters for $P_{\ell}^{\mathrm{DLNT}}(\kappa)$ in potentials $n=6$

\begin{tabular}{llllllll}
\hline$\ell$ & $C_{\ell}$ & $C_{\ell}{ }^{*}$ & $C_{\ell}^{\mathrm{WKB}}$ & $\kappa_{\ell}^{(1 / 2)}$ & $\kappa_{\ell}^{*}$ & $a_{\ell}$ & $b_{\ell}$ \\
\hline 1 & 0.465 & 0.42 & 0.641 & 1.247 & 0.845 & 1.25 & -0.35 \\
2 & $8.50 \times 10^{-3}$ & $8.5 \times 10^{-3}$ & $1.03 \times 10^{-2}$ & 2.542 & 2.05 & 1.00 & -0.12 \\
3 & $4.22 \times 10^{-5}$ & $4.0 \times 10^{-5}$ & $4.86 \times 10^{-5}$ & 4.129 & 3.52 & 0.825 & -0.05 \\
4 & $8.56 \times 10^{-8}$ & $8.0 \times 10^{-8}$ & $9.57 \times 10^{-8}$ & 5.975 & 5.31 & 0.730 & -0.035 \\
\hline
\end{tabular}

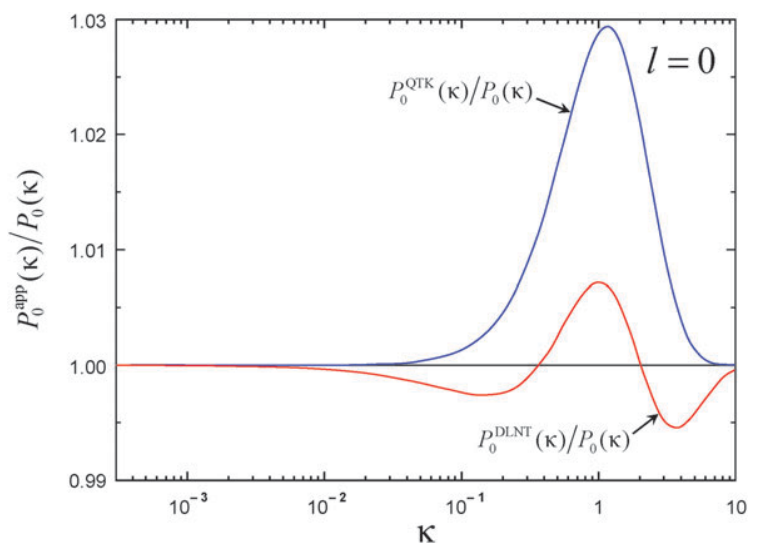

Fig. 7 As in Fig. 1, but for $n=6$.

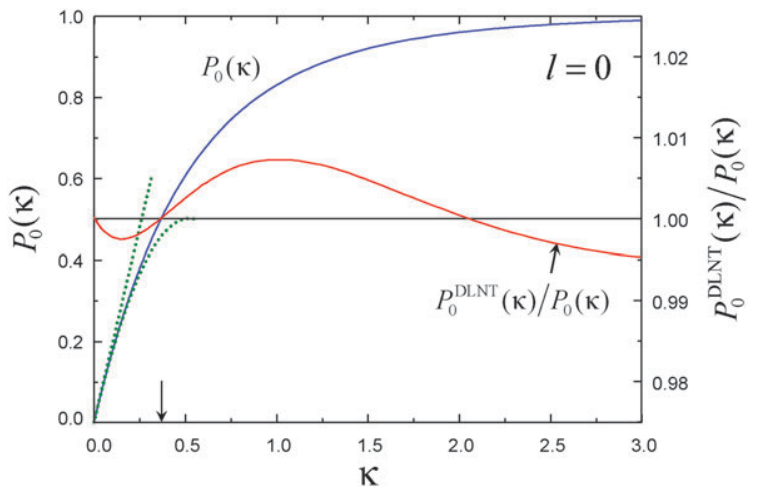

Fig. 8 As in Fig. 2, but for $n=6$

QTK formula works noticeably better here, especially for small $\kappa$. This is expected since the potential $\propto R^{-6}$ is more steep compared to that $\propto R^{-4}$, and therefore it is better simulated by an exponential drop, for which QTK expression is exact.

For $\ell=1-4$, the counterparts of eqn (19) and (21) retain the same form with different set of parameters. Table 2 lists the values of the relevant parameters. The plots of the capture probabilities and accuracy graphs for $\ell=0-4$ with $n=6$ are qualitatively similar to those for $n=4$, and are not given here.

\section{Conclusion}

The aim of this work was twofold: to study, for attractive isotropic $R^{-n}$ potentials ( $n=4$ and $n=6$ ), the performance of the Bethe-Wigner threshold laws for small wave vectors $k$ (and small probabilities $\left.P_{\ell}^{(n)}(k)\right)$ and to provide practically useful approximate analytical fits of $P_{\ell}^{(n), \text { app }}(\kappa)$ to numerical capture probabilities when $P_{\ell}^{(n)}(k)$ are not small.
For small $k$, we demonstrate the accuracy of the Bethe-Wigner analytical expression for the capture probabilities by comparing them with accurate numerical results. For medium $k$, the accurate graphs of $P_{\ell}^{(n)}(k)$ are supplemented with the plots of the ratio $P_{\ell}^{(n), \text { app }}(k) / P_{\ell}^{(n)}(k)$ that demonstrate the accuracy of the fitted $P_{\ell}^{(n), \text { app }}(k)$ which were forced to coincide with $P_{\ell}^{(n)}(k)$ when the latter equals $1 / 2$.

The results obtained serve for quick estimates of partial cross sections and rate coefficients for quantum capture, as well as for considering limitations in the application of the threshold relations. (We also note in passing that $P_{\ell}^{(4) \text {,app }}(\kappa)$ provides an approximation to the reference curves $\left.P(\lambda, \eta)\right|_{\eta=\ell}$ that lie on the two-dimensional surface $P=P(\lambda, \eta)$ in Fig. 1 of ref. 11, which represent the probabilities of capture of a charged particle by a stationary isotropically-polarizable dipole). We, therefore, recommend the use of eqn (11) and (23) for $l=0$ and $n=4$ and 6 , respectively. For $l=1-4$ on the other hand, eqn (19) is recommended with $g_{l}(\kappa)$ defined by eqn (18) and $H_{l}(\kappa)$ taken from eqn $(21)$ with the coefficients given in Table 1 and 2 for $n=4$ and $n=6$, respectively. The given approximate analytical expressions are of considerable practical use. They appear particularly useful in the modeling of electron attachment to polarizable neutral molecules (and the reverse detachment processes), see e.g. ref. 14-18, and may serve for describing the "quantum onset" of neutral-neutral reactions $(n=6)$ at low temperatures. ${ }^{1,12,13,19}$

\section{Acknowledgements}

Financial support of this work by EOARD (Grant Award No. FA8655-09-3001) is acknowledged.

\section{References}

1 E. I. Dashevskaya, I. Litvin, A. I. Maergoiz, E. E. Nikitin and J. Troe, J. Chem. Phys., 2003, 118, 7313.

2 C. E. Klots, Chem. Phys. Lett., 1976, 38, 61.

3 E. I. Dashevskaya, I. Litvin, E. E. Nikitin and J. Troe, Phys. Chem. Chem. Phys., 2008, 10, 1270 (in the Appendix of this article, $G_{l}(\kappa)=0.5+0.5\left[1+\tanh H_{l}(\kappa)\right]$ should be replaced by $\left.G_{l}(\kappa)=0.5\left[1+\tanh H_{l}(\kappa)\right]\right)$.

4 L. D. Landau and E. M. Lifshitz, Quantum Mechanics, Pergamon Press, Oxford, 1977.

5 M. J. Moritz, C. Eltschka and H. Friedrich, Phys. Rev. A: At., Mol., Opt. Phys., 2001, 63, 042102.

6 H. A. Bethe, Rev. Mod. Phys., 1937, 9, 69.

7 E. P. Wigner, Phys. Rev., 1948, 73, 1002.

8 H. Friedrich and J. Trost, Phys. Rep., 2004, 397, 359; Corrigendum: H. Friedrich and J. Trost, Phys. Rep., 2007, 451, 234.

9 M. Quack and J. Troe, Ber. Bunsen-Ges. Phys. Chem., 1974, 78, 240.

10 E. Vogt and G. H. Wannier, Phys. Rev., 1954, 95, 1190

11 E. I. Dashevskaya, I. Litvin, E. E. Nikitin and J. Troe, J. Phys. Chem. A, 2009, DOI: $10.1021 /$ jp901515e.

12 J. P. Hessler, J. Chem. Phys., 1999, 111, 4068.

13 J. Troe and V. G. Ushakov, J. Chem. Phys., 2008, 128, 204307. 
14 I. I. Fabrikant and H. Hotop, Phys. Rev. A: At., Mol., Opt. Phys., 2001, 63, 022706

15 H. Hotop, M.-W. Ruf, M. Allan and I. I. Fabrikant, Adv. Atom, Mol., Opt. Phys., 2003, 49, 85.

16 A. S. Dickinson, J. Phys. B: At., Mol. Opt. Phys., 2007, 40, F293.
17 J. Troe, T. M. Miller and A. A. Viggiano, J. Chem. Phys., 2007, 127, 244303.

18 J. Troe, T. M. Miller and A. A. Viggiano, J. Chem. Phys., 2009, 130, 244303.

19 A. S. Dickinson, J. Phys. B: At., Mol. Opt. Phys., 2007, 40, F237. 